\title{
INTELIGÊNCIA E DEFICIÊNCIA MENTAL NUM OLHAR VOLTADO PARA A COMPLEXIDADE
}

\section{Roberto Gimenez*}

ResumO: Atualmente, um tema que tem despertado a atenção e, por isso, tem sido motivo de debate e questionamento é a inteligência. Por exemplo, muitos termos como inteligência artificial, inteligência coletiva, inteligência emocional emergem no cenário internacional. Não há dúvida de que a discussão de aspectos relacionados à inteligência assume uma importância ainda maior ao se levar em consideração o problema da deficiência. O propósito do presente artigo é levantar alguns temas que consideramos pertinentes à discussão do conceito de deficiência, atribuindo uma especial ênfase às relaçôes da deficiência com a atuação dos profissionais de Educação Física.

\section{Visão tradicional de inteligência versus visão sistêmica de inteligência}

O estigma que acompanha o problema da deficiência em nossa sociedade tem contribuído para a elaboração de preceitos legais diferenciados para essa população, seja no âmbito Federal ou Estadual (vide p. e. documentos oficiais do Programa de Remoção de Barreiras ao Portador de Deficiência, emitido em 1992 pelo Ministério da Ação Social, e Programa Estadual de Atenção à Pessoa Portadora de Deficiência, promovido pelo Fundo Social de Solidariedade do Estado de São Paulo), e também tem levado profissionais especializados em diversas áreas a discutirem a natureza do problema.

Entre os temas que têm despertado maior interesse para discussão, encontram-se o diagnóstico e a classificação adotada para a deficiência. Amiralian (1995), referindo-se ao diagnóstico, enfatiza que, classicamente, este parece ter
PalaVRAS-CHAVE: Educação Física, complexidade, desenvolvimento humano, inteligência, deficiência mental.

*Professor dos Cursos de Educação Física do Centro Universitário Nove de Julho/ Universidade Cidade de São Paulo/Faculdade Ítalo-Brasileira; Coordenador de Educação Física do Centro de Apoio Acadêmico ao Deficiente (CAAD) da UNICID. Mestrando Membro do Laboratório de Comportamento Motor da Escola de Educação Física e Esporte da Universidade de São Paulo.

\section{$\mathrm{E}$}

C 
assumido um sentido restrito. O diagnóstico da deficiência mental, por exemplo, é com freqüência utilizado segundo o modelo médico ou psicométrico. No modelo médico, atribui-se demasiada ênfase a aspectos de ordem patológica e ao uso de instrumentos de medida que auxiliem o médico a confirmar suas conclusões. Por outro lado, o modelo psicométrico parte do pressuposto teórico da existência de características genéticas no comportamento, de ordem constitucional e imutáveis, utilizando-se de testes para medi-las, identificá-las e classificá-las. Desses testes, derivaram termos como idiota, imbecil, infradotado etc.

Embora mais recentemente o emprego desses termos tenha sido motivo de grande questionamento e debate, o que se evidencia é a utilização de outros, que ainda denotam um certo padrão estático de comportamento e relacionam a idéia da inteligência a algo que pode ser medido. Uma questão que se propõe é: Será que o nível de inteligência pode ser mensurado? Se isso for possível, então como fazê-lo?

$\mathrm{O}$ teste de Alfred Binet foi estabelecido com a intenção inicial de identificar crianças que apresentassem atraso no desenvolvimento, para que fosse possível realizar intervenção terapêutica adequada. Posteriormente, viu-se uma gradual aplicação desse teste para categorizar ou classificar os indivíduos, fato que se agravara com a emergência da primeira grande guerra. $\mathrm{O}$ que se verifica é que a idéia inicial do teste foi corrompida, sendo sugerido o seu emprego inclusive como um elemento para uma nova organização da sociedade americana. Entretanto, é interessante observarmos os muitos exemplos da vida cotidiana que corroboram a idéia de que a inteligência não pode ser categorizada a partir de um referencial tão rígido ou estático.

Considerando o contexto particular da educação especial e, mais especificamente, daquela relacionada ao indivíduo portador de deficiência mental, verifica-se que, durante muitos anos, o teste psicométrico foi considerado o referencial mais importante para a caracterização da deficiência, o que, sobretudo na última década, tem sido criticado por vários especialistas e docentes.

Em geral, prevalecia a visão de que deficiência mental não constituía um fenômeno simples, mas um problema complexo (Vieira de Souza, 1988; Strauss, 1988), resultante de muitas causas e sujeito a muitas categorizaçôes, nas quais a inteligência inadequada ou insuficientemente desenvolvida representa o denominador comum. Por outro lado, outras definições enunciam que a deficiência mental se refere a um funcionamento intelectual significativamente abaixo da média, que se manifesta ao longo do período de desenvolvimento e se caracteriza por uma 
inadequação da "conduta adaptativa" (Damasceno, 1992). É importante ressaltar que essa "conduta adaptativa" corresponde à capacidade de o indivíduo atingir os padrões esperados para sua faixa etária, no tocante à independência pessoal e responsabilidade social no contexto cultural em que se encontra. Não há dúvida de que considerar a capacidade de o indivíduo atender às demandas do seu meio social já representa uma evolução no que se refere à mera utilização de uma escala psicométrica. Todavia, existe a necessidade entender o que é a deficiência em si. Especialmente quando pensamos na questão da deficiência mental, é impossível que se avance na discussão sem questionar a concepção de inteligência prevalecente.

Até os dias de hoje, evidenciamos a utilização, em larga escala, de testes que possibilitam a mensuração da inteligência. Em outras palavras, conforme ressaltamos, a inteligência é vista como uma variável passível de ser medida a partir de testes. Esta é uma discussão pertinente à educação escolar de uma maneira geral. Evidentemente, o que pode ser perguntado é: Um aluno pode ser considerado deficiente por não apresentar um saber acadêmico?

Mais especificamente, esses problemas derivam de uma perspectiva de compreensão do ser humano reducionista e fragmentada. Muito provavelmente, o diagnóstico e a própria intervenção sobre a deficiência seriam modificados, caso o ser humano fosse analisado a partir de um referencial sistêmico (p.e. Weiss, 1967; Bertalanffy, 1977; Manoel, 1989). Por essa óptica, a relação entre parte e todo passaria a ser considerada. A limitação evidenciada numa parte, embora exerça certa influência sobre o todo, não pode ser generalizável a ele.

Concepções mais recentes sobre a inteligência (Gardner, 1993; Machado, 1996) vêm contrapor essa visão estática de inteligência, sugerindo que ela não pode ser medida e que deve ser entendida a partir da idéia de espectro, no qual as formas de manifestação das competências são múltiplas. O espectro de cada indivíduo sempre apresenta elementos, eixos, direções, relaçóes diagonais, especialmente vívidos e esmaecidos, sendo permanentes e inesgotáveis as possibilidades de aperfeiçoamento em praticamente todas as direções (Machado, 1996). A grande novidade dessa concepção, que se tem fortalecido, não é somente o seu caráter múltiplo, mas, sobretudo, a contraposição à medida com a idéia de espectro.

De acordo com Machado (1996), a inteligência deve ser considerada a partir de um espectro de competências, entre elas: (a) lingüística; (b) lógico-matemática; (c) corporal-cinestésica; (d) espacial; (e) musical; (f) interpessoal; (g) intra-pessoal; 
(h) pictórica.

A dimensão lógico-matemática tem sido regularmente considerada nos estudos de psicólogos, epistemólogos e até biólogos, como Piaget. Normalmente essa competência está associada à capacidade de estabelecer raciocínios dedutivos com a construção de cadeias causais, com a capacidade de solucionar problemas que lidam com números e objetos matemáticos.

A dimensão lingüística, assim como acontece com a lógico-matemática, também é bastante explorada por áreas como a psicologia e a pedagogia. Essa competência se expressa de modo peculiar no orador, no escritor, enfim, em todos os que lidam de maneira criativa com palavras.

No que diz respeito à competência corporal-cinestésica, é possível afirmar que ela é caracterizada pela capacidade de realizar movimentos, sem no entanto apresentar cadeias de raciocínios muito elaboradas nem expressá-los verbalmente.

A dimensão espacial da inteligência relaciona-se à competência para perceber e administrar adequadamente o espaço, à utilização adequada de mapas, de plantas e de representações em geral.

As competências interpessoal e intrapessoal estão intimamente relacionadas e correspondem, respectivamente, à capacidade de relacionar-se bem com os outros, de perceber seus humores, motivações e de sentir-se bem consigo mesmo, sabendo, por exemplo, administrar os próprios humores e os impulsos de agressividade.

A competência pictórica associa-se às manifestaçōes artísticas de diversas naturezas, como aquelas empregadas na confecção de desenhos e pinturas. Finalmente, a competência musical representa uma competência no seu estado puro, relacionada de maneira mais específica com a música.

Recorrendo a um panorama sobre a estrutura do sistema educacional e também da própria sociedade, torna-se nítida a hegemonia prevalecente das competências lógico-matemática e lingüística em detrimento das demais. No entanto, ao se priorizar excessivamente esse eixo, corre-se o risco de contribuir para a formação fragmentada de um ser humano, desenvolvendo apenas parcialmente suas potencialidades. Essencialmente, a questão da inteligência constitui um tema complexo. Temas complexos merecem ser abordados a partir de diferentes áreas (Morin, 1990) e, por isso, considera-se inadmissível conceber a inteligência a partir de um referencial unilateral, bem como orientar o processo de intervenção sobre 
o ser humano com base em única faceta de seu comportamento.

Nossa experiência como professores de educação física de indivíduos portadores de deficiência mental tem proporcionado alguns elementos para reflexão, sob um grau de esclarecimento mais amplo, a partir do conceito de inteligências múltiplas. Não são raros os casos de indivíduos portadores de deficiência mental, considerados pelas escalas de desenvolvimento cognitivo como pertencentes ao grupo de moderados, que são "experts" quando solicitados a executarem habilidades motoras relacionadas à modalidade esportiva do futebol. Sendo assim, é pertinente questionar: Ele é deficiente em quê?

A compreensão da inteligência a partir de seu caráter múltiplo, de espectro, vem contribuir para um melhor entendimento da natureza da deficiência. Se, por um lado, o indivíduo apresenta um determinado eixo ou a direção do eixo comprometida, por outro, apresenta condições de restabelecer o equilíbrio por meio de uma outra competência muito bem desenvolvida.

É fundamental destacar que essa visão sistêmica do ser humano e da deficiência tem sido negligenciada não somente pelo diagnóstico clínico, educacional, mas também pela própria intervenção pedagógica da Escola. A prática pedagógica do profissional de Educação Física tem refletido essa problemática. Essa temática é alvo de discussão na próxima seção.

\section{A visão tradicional da Educação Física versus a visão atual: no- vos horizontes}

Até meados da década de 80, não era comum encontrar-se o profissional de Educação Física trabalhando com indivíduos portadores de deficiência. Em que pesem as razões relacionadas às próximas tendências do mercado de trabalho, que indicavam um ramo de atuação muito mais direcionado para a área escolar, não há dúvida de que um fator crucial para dificultar esse envolvimento diz respeito ao próprio paradigma vigente na área da Educação Física.

A formação do professor de Educação Física era eminentemente norteada por procedimentos técnicos: o corpo de conhecimentos acerca do ser humano derivava, única e exclusivamente, das Ciências Biológicas, o que contribuiu para uma visão fragmentada do ser humano. 
*Designação atribuída à pratica pedagógica da Educação Física orientada para indivíduos portadores de deficiência.
Todavia, a partir dos anos 90, tem sido evidenciada uma expansão dos horizontes de atuação desse profissional, principalmente nos setores que extrapolam as dimensões da Escola, ou da Educação Formal. Essa tendência acabou por se tornar tão marcante que os próprios estabelecimentos universitários de ensino procuraram instrumentalizar-se com o objetivo de contribuir para a formação de profissionais mais bem preparados para atender às demandas e à concorrência presentes no mercado de trabalho.

Em meio a esse horizonte ampliado do mercado de trabalho, o profissional de Educação Física tem experimentado um contato cada vez maior com populaçóes diferenciadas, que fogem aos padrões tradicionalmente conhecidos. Entre esses grupos encontram-se, por exemplo, as crianças com problemas de asma nas academias e condomínios, os adultos obesos, hipertensos e coronarianos dos spas e clubes e os idosos em centros de convivência especializados. Outra área que passou a merecer consideração foi a da Educação Física Especial ou Educação Física Adaptada*, mais especificamente no que se refere à atuação com portadores de deficiência mental.

Pensando no trabalho com portadores de deficiência mental, um dos fatores que merecem atenção especial é a necessidade de um profissional que conheça efetivamente as características desses indivíduos. As peculiaridades inerentes ao comportamento das pessoas portadores de deficiências mentais implicam, muitas vezes, procedimentos pedagógicos diferenciados daqueles genericamente empregados nos indivíduos considerados normais.

A metodologia pedagógica convencional, empregada pela Educação Física, ainda deriva, em grande parte, de uma influência militarista que a área recebeu durante muito tempo (Marinho de Oliveira, 1983). Essencialmente, essa influência aliada à escassez de conhecimentos sobre a capacidade de os indivíduos portadores de deficiência realizarem movimentos tem constituído uma barreira à atuação do profissional de Educação Física.

De uma maneira geral, a prática pedagógica da educação física ainda está norteada por um ideal de indivíduo "normal". Esse enfoque se reflete na própria utilização de estilos de ensino tipicamente diretivos, tendo como base o modelo ou demonstração do professor. Outro impasse refere-se aos métodos empregados na avaliação que, em geral, levam em consideração esse modelo de "ideal" ou "perfeito". 
Parcialmente, essa maneira de compreender a deficiência se deve ao próprio método científico. Segundo Manoel (1996), a ciência busca identificar padrōes e regularidades na natureza. Uma vez detectados, busca-se explicá-los. Para ele, as ciências do comportamento, ao longo de sua história, têm adotado a estratégia de agregar dados de um número relativamente grande de sujeitos para evitar "erros" de interpretação, de acordo com os pressupostos básicos de estatística. A partir desse procedimento, procura-se revelar leis gerais que possam explicar o funcionamento dos sujeitos individualmente. Sendo assim, os resultados de pesquisa são generalizados a um indivíduo ideal e abstrato. É dessa concepção que surge a idéia de "normal" usada como referência para identificar, julgar e classificar o comportamento da pessoa portadora de deficiência.

$\mathrm{O}$ uso tradicional dessa metodologia nomotética pressupõe que seja importante identificar o indivíduo típico ou médio. A variabilidade intra e interindividual é normalmente esquecida ou eliminada em pesquisas com a população normal. A variabilidade é vista como algo negativo, o que, por nossa visão, consiste numa atitude errônea, não somente para a compreensão do comportamento da população normal, como também para o estudo do comportamento do indivíduo portador de deficiência, sobretudo quando consideramos que a investigação e a crescente compreensão do desenvolvimento humano têm mostrado que os padrōes de ação de toda pessoa representam um mosaico de interações e transações entre fatores biológicos, históricos e situacionais.

Conforme enfatiza Manoel (1996), ao se considerar a capacidade de o indivíduo portador de deficiência realizar movimento, é fundamental ter como base a noção de equivalência motora. Esse conceito pressupóe que uma mesma ação motora pode ser alcançada por meio de diferentes movimentos. Sendo assim, assumir que um portador de deficiência mental deve apresentar um padrão de movimento similar ao de um indivíduo normal reflete uma visão estática dessa deficiência. Muitas vezes, esse indivíduo pode ser capaz de realizar a mesma ação motora, recorrendo a outro tipo de movimento. É possível especular que a dimensão da competência corporal-cinestésica desse indivíduo extrapole a margem de alcance de um padrão em particular.

Essa perspectiva de análise sobre o comportamento humano e sobre o entendimento da deficiência encontra respaldo nos pressupostos de Sacks (1995), que 
sugere que uma doença, embora possa representar um fator de desordem para o indivíduo, leva-o também a buscar novos e diferentes estados de organização. Em outras palavras, a doença, assim como a deficiência, podem, paradoxalmente, ser geradoras de ordem. Sacks (1995) defende que indivíduos portadores de deficiência apresentam soluçôes tão criativas quanto as que um indivíduo considerado normal apresentaria.

Concorrem para uma visão desse tipo os pressupostos do pensamento complexo (p. e. Morin,1990), que sugerem que o alcance de níveis mais complexos implicam, necessariamente, a passagem por etapas de desorganização. Em outras palavras, estados de complexidade organizada podem emergir a partir de outros cuja base é a desordem.

No entanto, como muitas vezes se adota um critério absoluto para a análise do desempenho, o comportamento do indivíduo portador de deficiência é visto como rudimentar, precário ou mesmo ineficaz, concepção que, como vimos, necessita ser revista. Fundamentalmente, ao recorrer ao pensamento linear, a ciência, de maneira similar à cultura (Mariotti, 2000), acaba encontrando dificuldade de produzir modelos mentais que se diferenciem desse padrão e que, conseqüentemente, promovam maiores avanços.

Essa visão mais dinâmica do problema da deficiência, somada ao conceito de inteligências múltiplas, tem despertado interesse na área de Educação Física (p.e. Burton \& Davis, 1992; Bouffard et. al. 1998; Gimenez, 1999), bem como proporcionado elementos importantes para a discussão: O que é ser deficiente?

Uma maior reflexão sobre o comportamento do indivíduo portador de deficiência e, mais especificamente, do indivíduo portador de deficiência mental (levando-se em consideração essa concepção de inteligência) faz-se necessária, sobretudo, quando se toma como base a riqueza de alternativas proporcionadas pelo cérebro humano. Na realidade, acreditamos que deva prevalecer um olhar mais dinâmico e não rotulador para o entendimento e a caracterização dos indivíduos portadores de deficiência e também para a condução dos processos de intervenção pensados para essa população. Em especial, esse olhar deve nortear também a Educação Física, que surge como uma área com potencial para permitir a integração desse indivíduo na sociedade complexa. 
ABSTRACT: Nowadays, one of the subjects that has aroused attention is the intelligence and that's why, it has been a reason for debates and questioning. For example, many terms like artificial intelligence, collective intelligence and emotional intelligence emerge in the international scenery. There is no doubt that, the discussion related to intelligence receives a bigger importance if we consider the handicap problem. The purpose of this essay is to raise some aspects that we think that are pertinent to the discussion of the handicap concept, giving a special emphasis to the relation between handicap and the physical education professionals.

\section{REFERÊNCIAS BIBLIOGRÁFICAS}

Amiralian, M. L. T. O Diagnóstico da Dehâencia Mental Boletim de Psicologia v. $1996, \mathrm{XLV}$, n. 102 , p. 35-44.

Bertal anffy, L.V. (1977) Teoria Geral dos Sistemas. Petrópolis, Vozes.

Burt on, A \& Davis, W. E. (1992). Optimizing the envolvement and performance of children with physical impairments in movement activities. Pediatric Exercise Science, 4, 238-248.

Bouffard, M.; St rean, W.B. \& Davis, W. (1998). Questioning our phylosophical and methological research assumptions: Phychological perspectives. Adapted Physical Activity Quaterly, [15], p.250-268.

Damasceno, L. G. (1992). Natação, Psicomotricidade e Desenvolvimento. Brasília, Secretaria dos Desportos da Presidência da República.

Fundo Social de Sol idariedade. Programa Estadual de Atenção à Pessoa portadora de Deficiência, São Paulo, 1994.

Gardner, H. (1993). Multiple intelligences. New York, BasicBooks.

Gimenez, R. (1999). O profissional de Educação Física na Equipe Multidisciplinar: Caminhos a serem trilhados na busca da Identidade, [4], 4, p. 56-58.

Machado, N. J. (1996). Epistemologia e Didática. São Paulo, Cortez.

Manoel, E. de J. (1996) O Estudo do Comportamento Motor da Pessoa Portadora de Deficiência: Problemas e Questōes. Revista Brasileira de Saúde Escola, [4], 4, 11-21.
KEY WORDS: Physical Education, complexity, human development, intelligence, mental handicap. 
(1989). Desenvolvimento do Comportamento Motor Humano: Uma abordagem Sistêmica. Dissertação (Mestrado). São Paulo: Universidade de São Paulo.

Marinho de Ol iveir a, V. (1983) Educação Física Humanista. Rio de Janeiro, Ao Livro Técnico.

Minist ér io da Ação Social. Política Nacional de Integração da Pessoa Portadora de Deficiência, Brasília, 1992.

Mariott i, H. (2000) Cognição, Solidariedade e o Novo Autoritarismo. Eccos, [1], 2, p.27-43.

Mor in, E. (1990) Ciência com Consciência. Mira Sintra: Europa-América.

Sher rill, C. (1988). Leadeship Traning in adapted phisical education. Champaign, Human Kinetics Books.

Sacks, O. (1995). Um Antropólogo em Marte. Tradução de Bernardo de Carvalho. São Paulo, Cia das Letras.

St rauss, R. (1988). Motor Behavior and Learning: A multidisciplinary practicum for undergraduate students. In Sherrill, C. (ed.). Leadership Training in Adapted Phisical Education. Chapaign, Human Kinetcs Books, p.329-332.

Vieir a de Souza, J. (1988). A equipe Multidisciplinar no diagnóstico e orientação da deficiência mental. Fundação do Bem-estar do menor.

Weiss, P. (1969). Living System s: Determ inism stratified. In A Kostier \& J. Sm iTHIES (EDS.), Beyond reductionism. London, Hutchison \& Co. 